\title{
Aplicación de Ventana Pericárdica Subxifoidea en Traumas de Tórax Abiertos en Pacientes de Sala de Urgencias del Centro de Emergencias Médicas
}

\author{
Anna Fiorella Daiub Alviso, Marcos Federico Mieres Romero, Romina Elizabeth Galeano Patiño
}

\section{RESUMEN}

Objetivos: Determinar la frecuencia de la aplicación diagnóstica de la ventana pericárdica en pacientes con traumatismos torácicos abiertos (TTA) del Centro de Emergencias Médicas (CEM) y; comparar la exactitud de la ventana pericárdica subxifoidea (VPS) en el diagnóstico de patologías post traumáticas torácicas respecto a métodos no invasivos (E copericardio - EP).

Materiales y métodos: Se realizó un estudio observacional analítico retrospectivo que abarca los meses de Enero a Diciembre del año 2010 y los meses de Enero a Mayo del año 2011. Se analizaron durante el periodo de estudio un total de 26 pacientes con traumatismo torácico abierto y aplicación de VPS. Se acudió a los registros de sala de Cirugía del CEM, de manera que el estudio se sustenta en información de fuente secundaria, utilizándose métodos de representación estadística.

Resultados: De 322 casos de trauma torácico abierto, 26 casos con ecopericardio indeterminado $(8,07 \%)$ fueron analizados. De tales casos y utilizando como método diagnóstico secundario la VPS, 22 resultaron positivos $(84,61 \%)$ evitando lesiones cardíacas severas con posible derivación fatal en éstas y toracotomías innecesarias en las restantes 4 personas (15,39\%).

Conclusión: La aplicación diagnóstica de Ventana Pericardica Subxifoidea (VPS) es la forma más sensible y específica de determinar lesiones cardiacas con compromisos vitales ante una prueba diagnóstica no invasiva (ecopericardio) con resultado no concluyente.

Palabras clave: Trauma de tórax abierto, Ventana pericárdica, Ventajas.

\section{ABSTRACT}

Objectives: Determine the frequency of the application of a diagnostic pericardial window in patients with open chest trauma (OCT) of the E mergency Medical Center (E MC) and, to compare the accuracy of the subxiphoid pericardial window (SPW) in the diagnosis of posttraumatic thoracic pathologies vs noninvasive methods (E copericardium-EP).

Materials and methods: We performed a retrospective observational study covering the months of J anuary to December of 2010 and the months of J anuary to May of 2011. A total of 26 patients with open chest trauma and application of VPS were analyzed during the study period. The records from the ECM surgery ward were analyzed; the study is based on information from secondary sources, using methods of statistical representation.

Results: Out of 322 cases of open chest trauma, 26 cases with undetermined ecopericardium $(8.07 \%)$ were analyzed. In such cases and using as a secondary diagnostic method VPS,
22 were positive (84.61\%) avoiding severe heart damage with possible fatal bypass of these and unnecessary thoracotomy in the remaining four people (15.39\%).

Conclusion: The diagnostic application of subxiphoid pericardial window (SPW) useful to determine cardiac lesions with vital compromise vs a non-invasive diagnostic test (ecopericardio) with inconclusive results.

Keywords: O pen chest trauma, Pericardial window, Advantages.

How to cite this article: Alviso AFD, Romero MFM, Patiño REG. Aplicación de Ventana Pericárdica Subxifoidea en Traumas de Tórax Abiertos en Pacientes de Sala de Urgencias del Centro de Emergencias Médicas. Panam J Trauma Critical Care Emerg Surg 2012;1(2):88-91.

\section{Source of support: Nil}

\section{Conflict of interest: None}

\section{INTRODUCCIÓN}

Las lesiones cardiacas traumáticas son causadas por lesión penetrante 0 no penetrante. Las lesiones penetrantes muy a menudo se deben a heridas por arma blanca 0 arma de fuego, y el sitio de entrada suele ser evidente. L as lesiones no penetrantes muy a menudo se presentan durante accidentes en vehículos motorizados, sea a consecuencia de una lesión por desaceleración rápida o por el impacto del tórax contra el volante, y conllevan una lesión cardiaca importante aun cuando no haya signos externos de traumatismo torácico. ${ }^{1}$ Dentro de las complicaciones más frecuentes del traumatismo torácico penetrante, se encuentra la posibilidad de lesión cardiaca con acumulación de sangre dentro del espacio pericárdico, conocido con el nombre de taponamiento cardiaco, que deriva al aumento de la presión intrapericárdica que origina aumento de la presión venosa y colapso circulatorio. El aumento de presión intrapericárdica ocurre cuando hay un incremento en forma rápida del líquido pericárdico por arriba de 60 a $80 \mathrm{ml}^{2}$

En condiciones normales puede haber hasta $50 \mathrm{ml}$ en el espacio pericárdico. ${ }^{2}$

En caso de derrame de instalación lenta, se puede estirar sin aumentar la presión intrapericárdica y pueden acumularse hasta 2 litros en estas condiciones.

Por ecografía la cantidad mínima detectable es de $15 \mathrm{~mm}$. Los derrames pequeños se los observa en sístole.

Paper presented in Student Research Competition at the 24th Annual Congress of SPT in Asuncion, Paraguay on November 2011. 
Existen situaciones que simulan extravasación de líquido al pericardio:

- Fibrosis

- Tejido neoplásico

- Grasa epicárdica

Para acontecimientos en que registre heridas de trauma de tórax abierto, uno de los métodos diagnósticos desarrollados es la ventana pericárdica, que se define como procedimiento que se realiza en casos donde se presenta acumulación excesiva de líquido al rededor del pericardio, con posibilidades de derivación a taponamiento cardiaco.

Este método diagnóstico fue descrito por L arrey en 1801. 'Todo paciente que presente una herida penetrante en el área comprendida por debajo de las clavículas, arriba de los márgenes costales y por dentro de la línea medio clavicular y epigastrio debe considerarse como herida cardiaca hasta no demostrar lo contrario'. Esta técnica evalúa la presencia de sangre en el pericardio, es rápida, segura y simple. ${ }^{3}$

Sea cual sea la causa, el tratamiento es la evacuación del líquido pericárdico y para fines de diagnóstico, en caso de que sea necesario, se determina la realización de una ventana pericárdica para toma de biopsias del pericardio y análisis del líquido. El procedimiento más usado es la ventana pericárdica subxifoidea, Para la creación de una ventana pericárdica subxifoidea se efectúa una incisión sobre el apéndice xifoides de $10 \mathrm{~cm}$ en dirección caudal. Se separa y se diseca el apéndice xifoides de las fibras de inserción del músculo recto anterior del abdomen, sujetándolo con una pinza y desplazándolo en sentido cefálico. Desplazando el tejido adiposo por debajo del apéndice y con disección roma se accede al pericardio que se sujeta con una pinza deA Ilis. Se realiza una incisión de $1 \mathrm{~cm}$ sobre el pericardio con cuidado de no lesionar el epicardio. Ventana pericárdica.

\section{OBJETIVOS}

- Determinar la frecuencia de la aplicación diagnóstica de la ventana pericárdica en pacientes con traumatismos torácicos abiertos (TTA) del Centro de Emergencias M édicas (CEM).

- Comparar la exactitud de la ventana pericárdica subxifoidea (VPS) en el diagnóstico de patologías post traumáticas torácicas respecto a métodos no invasivos (Ecopericardio - EP).

\section{MATERIALES Y MÉTODOS}

Este estudio retrospectivo descriptivo se llevó a cabo en el Centro de Emergencias M édicas 'Dr M anuel Giagni' de A sunción, Paraguay, comprendiendo un período de 17 meses desde Enero de 2010 a M ayo de 2011.
Los expedientes clínicos y libros de registro de cirugía de pacientes constituyeron la principal fuente de información, de tipo secundario, y los datos fueron seleccionados por instrumento estadístico tipo cuestionario, constituidos por criterios de relevancia para diagnostico de patología cardiaca post traumática mediante método de Ventana Pericárdica Subxifoidea, incluyendo en esta herramienta el grupo etario, mecanismo de lesión, tiempo de auxilio y métodos de diagnósticos tanto primarios como secundarios, procedimientos quirúrgicos posteriores y resultado del procedimiento diagnóstico.

\section{HERRAMIENTAS EPIDEMIOLÓGICAS}

Basados en los datos obtenidos por método estadístico aplicado, se registran resultados en cuadros y gráficos según cruce de variables estudiadas representada tanto en porcentajes como en frecuencias y números absolutos registrados durante a medición.

\section{RESULTADOS}

De un grupo de individuos estudiados de 322 con registro de ingreso por trauma torácico penetrante durante el periodo de tiempo correspondiente a Enero de 2010 a Mayo de 2011, se seleccionaron a 26 (8,07\% de población) pacientes con ecopericardio indeterminado, sometidos a método diagnóstico por VPS, en su totalidad correspondientes al género masculino, distribuyéndose en los siguientes grupos etarios: $17-20$ años (30,77\%), $21-25$ años $(38,46 \%)$ y de 26 a 40 años (23\%), de 40 años en adelante (7,7\%) (Cuadro 1).

\begin{tabular}{|cc|}
$\begin{array}{c}\text { Cuadro 1: Casos positivos y negativos diagnosticados } \\
\text { por VPS }\end{array}$ & Resultado \\
\hline № de casos & $(+)$ \\
24 & $(-)$ \\
\hline
\end{tabular}

Se pone de manifiesto claramente que la población mayoritariamente expuesta a eventos traumáticos torácicos es la de adul tos jóvenes, teniendo como pico de lesiones al grupo de personas entre 21-25 años, seguidos por aquellos de entre 17-20 años, población económicamente activa adulta joven. Con una media de edad correspondiente a: 24,5 años, una moda de 22 y 26 años y una mediana de 13,5 . (V éase Gráfico 1: Distribución de V PS en personas de sexo masculino según rango etario).

B asados en mecanismos de lesión, los datos se registran como sigue: 2 eventos por arma de fuego (7,7\%), los restantes 24 por arma blanca (92,3\%). (V éase Gráfico 2: Distribución de pacientes sometidos a VPS según mecanismo de lesión). 


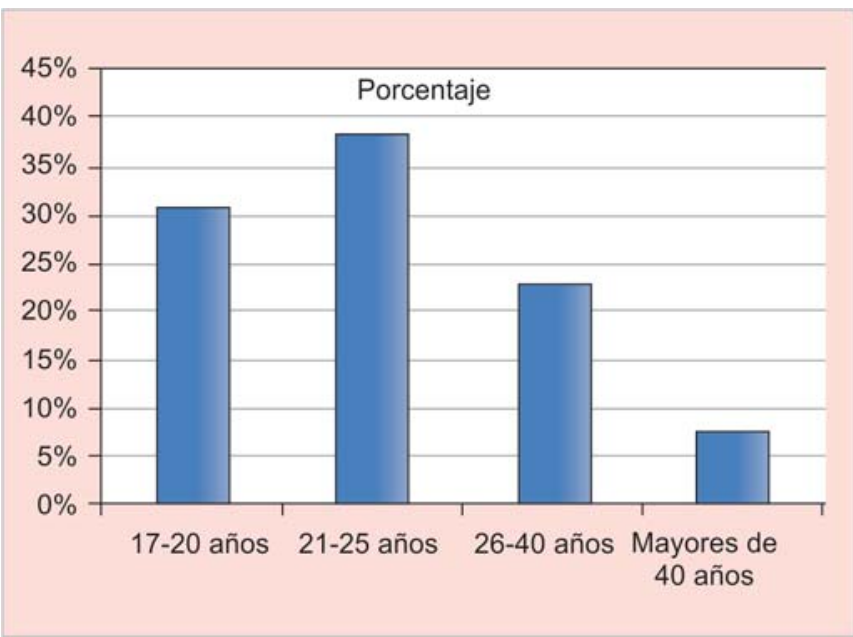

Gráfico 1: Distribución de VPS en personas de sexo masculino según rango etario

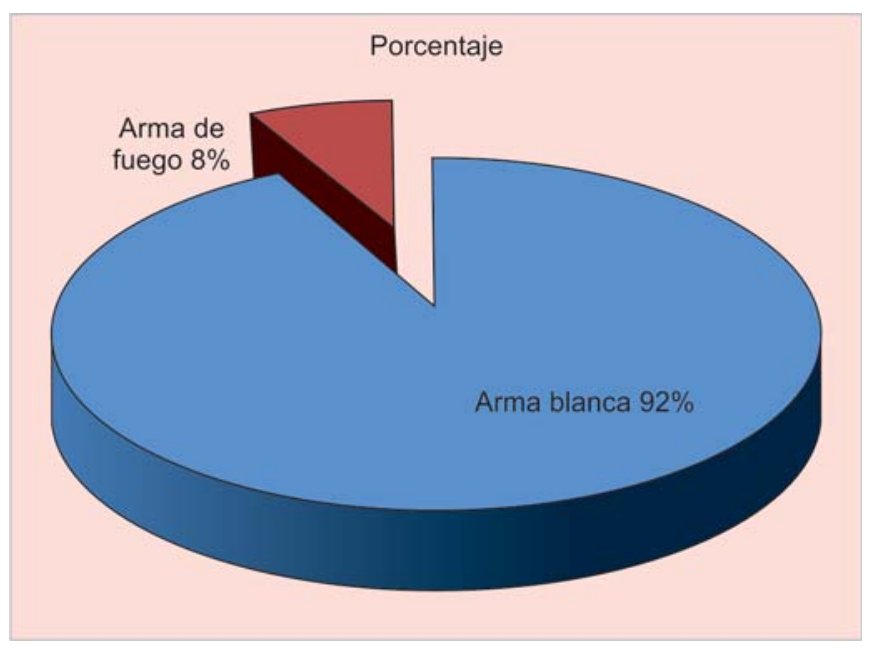

Gráfico 2: Distribución de pacientes sometidos a VPS según mecanismo de lesión

Con respecto a la procedencia, el $75 \%$ de los pacientes registra el evento traumático en zonas urbanas aledañas a la capital, con un tiempo de auxilio de entre 30 minutos y 1 hora, y $25 \%$ proviene del interior del país, con tiempos de auxilio mayores a una hora, siendo el máximo de 2 horas 50 minutos, con un promedio de tiempo de 51 minutos.

Coincidiendo con la coyuntura social actual, se manifiesta mayor frecuencia de eventos violentos dentro de zonas urbanas del país. (V éase Gráfico 3: Distribución pacientes con V PS según lugar de evento traumático).

La toracotomía anterolateral izquierda es el procedimiento quirúrgico más frecuentemente aplicado luego de un diagnóstico positivo por VPS.

$L$ as heridas penetrantes de corazón deben sospecharse en pacientes con lesión torácica anterior sobre el área precordial, epigastrio y mediastino superior (Sauer y M urdock).

Según manual de procedimientos vigente en CEM, Ios procedimientos diagnósticos se basan según la presentación

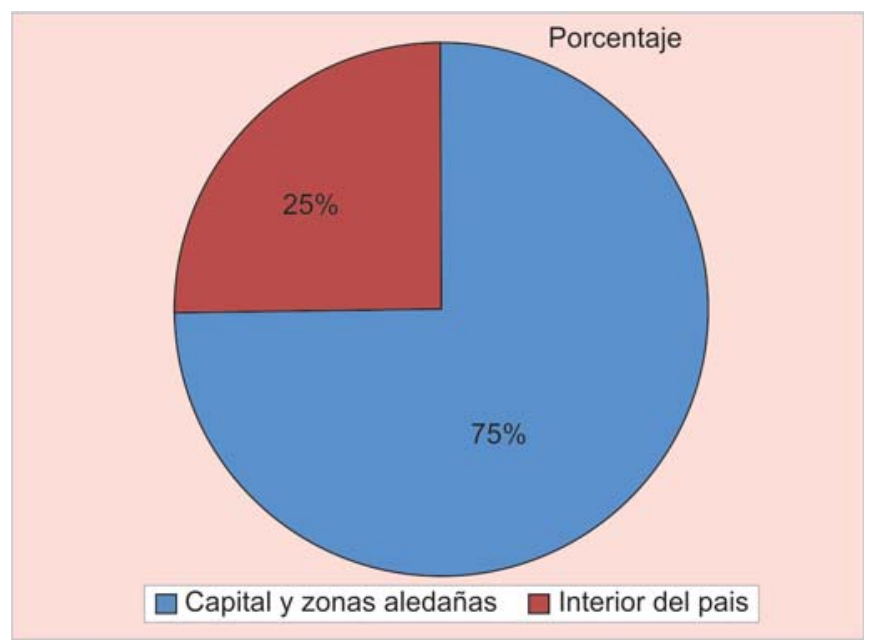

Gráfico 3: Distribución pacientes con VPS según lugar de evento traumático

clínica del paciente, estabilidad hemodinámica, tipo de lesión y rapidez de la asistencia, teniendo prioridad como método diagnóstico el ecopericardio, la V PS se realiza ante pacientes hemodinámicamente estables, con tiempo de progreso de lesión escaso a moderado y ecopericardio indeterminado, en cuanto a las toracotomías, predominan como procedimiento diagnóstico ante pacientes con hemodinamia comprometida y signos clínicos de alteración cardiaca.

\section{DISCUCIÓN}

Durante el estudio, se ha encontrado una frecuencia de aplicación de VPS de 26/322, es decir FRP $=8,07 \%$. La VPS ha demostrado un $100 \%$ tanto de sensibilidad como de especificidad, visto que todos los pacientes con VPS (+) presentaron lesiones cardiacas de diversa índole y localización, y aquellos con VPS (-) no desarrollaron complicaciones asociadas a lesiones cordiales. Del mismo modo se halló en este estudio que la eficacia relativa porcentual del Ecopericardio es de 91,93\%, es decir, diagnosticó correctamente lesión cardíaca traumática 0 su ausencia en 296 de 322 casos. Comparativamente, el parámetro anterior aplicado a la VPS arrojó un resultado de $100 \%$, es decir, diagnosticó correctamente la lesión o su ausencia en 26 de 26 casos posibles y por tanto consideramos que se mantiene como el Gold Standard para fines diagnósticos en eventos de trauma de tórax penetrante.

\section{REFERENCIAS}

1. A nthony $F$, et al. Harrison. Principios de M edicina Interna. 17a Edición 2009.

2. http://www.medsalud.com.ar. Taponamiento cardíaco: M edline Plus enciclopedia médica.

3. Ventana pericardica subxifoidea un método rápido y seguro para descartar lesión cardiaca en casos de heridas precordiales y transmediastinales en pacientes hemodinámicamente estables. Jorge Rodríguez-B endaña, Francisco Ayes-Valladares 1999A bril. 
Aplicación de Ventana Pericárdica Subxifoidea en Traumas de Tórax Abiertos en Pacientes de Sala de Urgencias

ACERCA DE LOS AUTORES

\section{Anna Fiorella Daiub Alviso}

(Correspondencia)

Estudiante de Tercer A ño de Medicina, Facultad de Ciencias de la Salud, U niversidad Católica 'Nuestra Señora de la A sunción' e-mail: fiodaiub_777@ hotmail.es

\section{Marcos Federico Mieres Romero}

Estudiante de Segundo A ño de M edicina, Facultad de Ciencias de la Salud, Universidad C atólica 'N uestra Señora de la A sunción'

\section{Romina Elizabeth Galeano Patiño}

Estudiante de Tercer A ño de Medicina, Facultad de Ciencias de la Salud, Universidad Católica 'N uestra Señora de la A sunción' 